\title{
Exploration and Practice of a New Formula for Calculating the Degree of Freedom
}

\author{
Yangxiaochi ${ }^{1}$ \\ ${ }^{1}$ School Of Engineering \&Technology ,Xi'an Fan Yi University, Xi'an 710105,China
}

\begin{abstract}
The current "structural mechanics" in the textbook, the more sub items in the formula of calculating degrees of freedom, is not conducive for the students to master the understanding and calculation of degree of freedom concept. After a few years of teaching, the new calculating formula of degree of freedom for the system is put forward, and the old formula is verified by two examples. The result shows that the new calculating formula and the current mainstream material formula calculation results are consistent, and the new formula is more helpful for students to understand the physical meaning of the calculation of degrees of freedom.
\end{abstract}

\section{INTRODUCTION}

In structural mechanics, in order to study whether the system can be used as a structure, it is often necessary to determine the degree of freedom of the system. On the one hand, if the degree of freedom of a system is 0 , the system is a geometrically stable system, namely, under the action of a load, the system can maintain the geometric shape and position without rigid body movement, which can be used as a structure; On the other hand, if the degree of freedom of a system is $>0$, the system is a geometrically unstable system, that is, under the action of a load, its geometry and position will change, which cannot be used as a structure.

The degree of freedom of the system is equal to the sum of the degrees of freedom of all constrained objects in the system minus the sum of all necessary constraints within the system. However, in the system, it is difficult to identify the necessary constraints under normal circumstances. Thus, the degree of freedom of the system is generally difficult to solve in most cases.

For this reason, in order to facilitate the study, we put forward the concept of computational freedom, which means the sum of freedom degree of all constrained objects in the system subtracts the sum of all constraints, and all the constraints include necessary constraints and redundant constraints.

Li Quanyong ${ }^{[4]}$ proposed a calculation formula for calculating the degree of freedom with a node as a basic unit to consider the degree of freedom. It is designed to calculate the truss structure consisting of two-force bar and extend this formula to a system of non-two-force bar. Through the example calculation, it is found that the formula has the same effect as the calculation formula of the freedom of the mechanism as the basic unit. Besides, compared with the conversion from the latter to the single hinge, the numbers of the independent nodes, members and struts in the former are simpler and more effective, which are less prone to errors and easy to grasp.Guo Weidong et al. ${ }^{[6]}$ proposed a new method for calculating the degrees of freedom of plane mechanisms based on an in-depth analysis of existing problems in the calculation of degrees of freedom of planar mechanisms in textbooks such as Theory of Mechanical and Mechanisms and Fundamental to Machine Design. By introducing new concepts of real-constraint higher pair, redundant-constraint higher pair, full- constraint lower pair, and half-constraint lower pair, the existing problems in the calculation of the degree of freedom of the planar mechanisms are solved, which is helpful for making the formula for calculating of plane mechanisms more rigid and scientific.Zhang Junde ${ }^{[10]}$ established the second type of general formula for calculating the degree of freedom of the rod system and verified it. Through the comparative study and application of calculation formulas for new and old degrees of freedom of institutional structure, Cai Hanzhong et al. found that the new formula and the K-formula are the best formulas for calculating the degrees of freedom of plane and space mechanisms. Besides, they also pointed out that the new formula is applicable to all structural mechanics degrees of freedom calculation, while the K's formula is only applicable to some parts.

In the daily teaching work, it is found that the physical meaning of the calculation formula of the computational degree of freedom in the current textbook of structural mechanics is not clear, which leads to a poor understanding of the students. Therefore, this paper proposes a new formula for calculating the degree of freedom. 


\section{Formulas For Calculating Degrees Of Freedom In Mainstream Structural Mechanics Textbooks}

\subsection{The formula that use rigid sheet as constrained object and use hinges and support chain rods as constraints}

$$
W=3 m-2 h-r
$$

In the formula, $\mathrm{W}$ represents the computational freedom of the system;m represents the number of rigid sheet pieces in the system; $h$ represents the number of single hinges in the system; $r$ represents the number of support chain rods.

\subsection{The formula that use hinges as constrained objects and use chain rods, hinges, and support chain rods as constraints}

$$
W=2 j-b-r
$$

In the formula, $\mathrm{W}$ represents the degree of freedom of the calculation of the system; $j$ represents the number of hinge points in the system; b represents the number of chain rods in the system; $r$ represents the number of support chain rods.

The above two formulas have clear concepts and the calculations are relatively simple, but students have the following deficiencies in understanding:

For formula 1, the non-supported chain rods are grouped into the rigid sheet and the support chain rods are listed separately. For students, a support chain rod can only be used as a support chain rod, while the non support chain bar is a stereotype of rigid sheet. In fact, the support chain link can also be regarded as rigid sheet. We know that in the analysis of geometric structures, under the premise of not considering the material deformation, a beam, a column, a geometrically uniform whole can be regarded as a rigid sheet. Therefore, the support chain bar can also be regarded as rigid sheet when it is considered as a constraint and it is just rigid sheet when regarded as a constrained object.

\section{The concepts and methods of computational freedom proposed in this paper}

Method 1: The system is regarded as consisting of a number of rigid sheets that are subject to the constraints of the hinge.

That is, the formula that regards all the chain rods (rigid sheet) in the system as the constrained objects and the hinges as the constraints.

Since the material deformation is not taken into account, we know that in the analysis of the geometrical composition of the planar system, a chain bar is a rigid sheet and the degree of freedom of a rigid sheet is 3 ; thus, single hinge is equivalent to two constraints. According to the definition of computational freedom, the computational freedom is equal to the sum of degrees of freedom of the constrained object in the system minus the sum of all constraints in the system. The formula for calculating the degree of freedom is as follows:

$$
W=3 m-2 h
$$

In the formula, $\mathrm{W}$ represents the computational freedom of the system; $m$ represents the number of rigid sheets in the system, which includes all the number of links in the system and support chain link. H represents the number of single hinges in the system, which also includes a hinge that is connected to the base.

Method 2: The system is regarded as consisting of a number of nodes that are bound by chain links.

That is, the formula that uses all hinges in the system as constraint objects and chain links as the constraints.

We know that a particle has two degrees of freedom in the plane and a chain bar is equivalent to a constraint. According to the definition of the computational freedom, the formula for calculating the degree of freedom is:

$$
W=2 j-b
$$

In the formula, $\mathrm{W}$ represents the degree of freedom of the system; $\mathrm{J}$ indicates the number of hinge points in an excluded system that is directly connected to the base, because the hinge connected to the base has no degree of freedom. That is, the degree of freedom is zero, and therefore it is excluded. B indicates the number of links in the system, which includes chain link and the support chain link in the upper system.

It can be seen that the above two formulas for calculating the degree of freedom have clear concepts and few parameters. In formula (3), considering the mechanical properties of the rods only, all the rods (including the support chain rods) are regarded as rigid sheets regardless of the actual use of the rods so that the students can deeply understand the meaning of the rigid sheets; In formula (4), regardless of the use of the link bar, the hinge point is regarded as a particle and a constraint object, as well as the link bar is regarded as a constraint.

\section{The application of examples}

\section{Example 1: Calculate the degree of freedom of the system shown in Figure 1(a).}

Solution: The method in the current textbook (Formula 1).

Take the rigid sheet as the constraint object and the hinge and the support chain rod as the constraints.

According to the physical meaning of formula (1), the rigid sheet only includes the rods in the upper system. In other words, rods $\mathrm{AB}, \mathrm{BC}, \mathrm{CD}, \mathrm{DE}, \mathrm{EF}, \mathrm{FA}$, and $\mathrm{BF}$ are rigid sheets. The number of tablets is $\mathrm{m}=8$.

The single hinge number of each hinge is shown in Fig. 1(b), and the single hinge number of the system is $\mathrm{h}=2+2+1+1+3+1=10$.

$\mathrm{AG}, \mathrm{AH}$, and EI are the supporting links, and the number of supporting links is $\mathrm{r}=3$.

Put $\mathrm{m}=8, \mathrm{~h}=10, \mathrm{r}=3$ into the formula $\mathrm{w}=3 \mathrm{~m}-2 \mathrm{~h}-\mathrm{r}$, then $\mathrm{w}=3 \times 8-2 \times 10-3=1$ is obtained, which means the degree of freedom of the system is 1 .

The method of this article ( Formula 3):

All the rods (including the support chain rods) in the system are regarded as constraints objects--- rigid sheets as well as all hinges (including hinges directly connected 
to the foundation) are regarded as constraints in the method of Formula 3.

According to formula 3 , there are three more rigid pieces in Fig. 1(a), namely the support chain members are $\mathrm{AG}, \mathrm{AH}$, and $\mathrm{EI}$, and the number of rigid pieces is $\mathrm{m}=11$.

Compared with the method of formula 1 , when the method of formula 3 is used, three hinges of hinge $G$, hinge $\mathrm{H}$, and hinge $\mathrm{I}$ are added. At the same time, the number of single hinges of hinge $A$ and hinge $E$ has changed, and the number of single hinges of each hinge is shown in Fig. 1(c).

Therefore, the single hinge number of the system is $\mathrm{h}=2+2+12+1+3+3+1+1=16$ and the system's single hinge number is $\mathrm{h}=16$.

Besides, the number of single hinges of hinge A and hinge $\mathrm{E}$ is changed, and the number of single hinges of each hinge is as shown in Fig. 1(c). Thus, the single hinge number of the system is $\mathrm{h}=2+2+12+1+3+3+1+1=16$. The system's single hinge number is $\mathrm{h}=16$.

Put $\mathrm{m}=11, \mathrm{~h}=16$ into the formula $\mathrm{w}=3 \mathrm{~m}-2 \mathrm{~h}$. Then $\mathrm{w}=3 \times 11-2 \times 16=1$ is obtained, which means the degree of freedom of the system is 1 .

Example 2: Calculate the degree of freedom of the system shown in Figure 2.

Solution: The method in the current textbook (Formula 2).

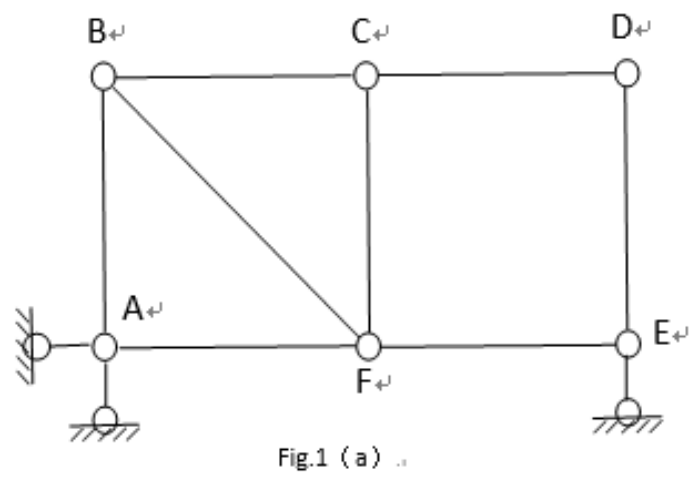

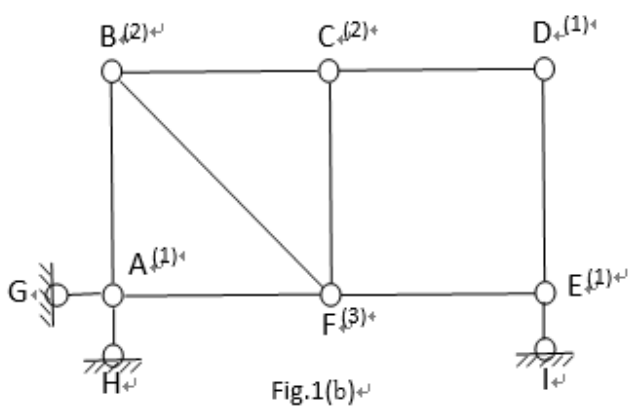

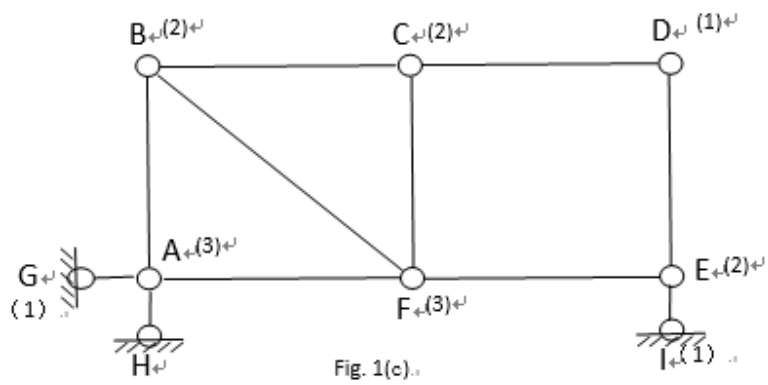

Formula 4 treats the hinge as a free body, that is, a constraint object, with the chain bar and the support chain bar in the upper system as constraints.

As can be seen from Fig. 2, there are six hinges in the upper structure of the system, namely hinges A, B, C, $\mathrm{D}, \mathrm{E}$, and $\mathrm{F}$. The number of hinge points in the system is $\mathrm{j}=6$.

Meanwhile, there are six chain links in the system, namely the chain links $\mathrm{AB}, \mathrm{BC}, \mathrm{CD}, \mathrm{DE}, \mathrm{EF}, \mathrm{FA}$, thus the number of chain links in the system is $b=6$.

Put $j=6, b=6, r=6$ into the formula $w=3 m-2 h$. Then $w=2 \times 6-6-6=0$ is obtained, which means the degree of freedom of the system is 0 .

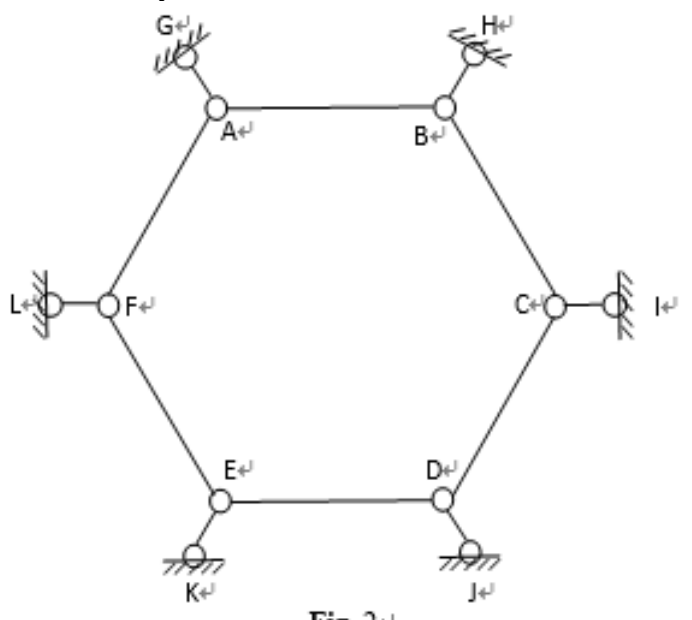

Fig. 2

The method of this article (Formula 4):

Formula 4 treats the hinge as a free body, that is, a constraint object and a chain bar as a constraint. As can be seen from Fig. 2, there are 12 hinges in the system, namely hinges A, B, C, D, E, F, G, H, I, J, K, and L.

However, hinges G, H, I, J, K, and L are directly connected to the foundation, and the degree of freedom is zero. If the degree of freedom is excluded, the number of hinge points in the system is $\mathrm{j}=6$. There are 12 chain links in the system, namely, the links $\mathrm{AB}, \mathrm{BC}, \mathrm{CD}, \mathrm{DE}, \mathrm{EF}, \mathrm{FA}$, 
AG, BH, CI, DJ, EK, and FL. Thus, the number of links in the system is $b=12$.

Put $J=11, b=12$ into the formula $w=2 j-6$. Then $2 j-b=2$ $\times 6-12=0$ is obtained, which means the degree of freedom of the system is 0 .

\section{Conclusions}

Through the above two examples, using the method of this article and the method of the current textbook, we obtained the same results of freedom of the system. Meanwhile, the former has a clear concept and a simple calculation process, which are easy for students to understand the concepts of rigid sheet and degree of freedom. The calculation formula of the computational freedom proposed in this paper is the author's summary and exploration in the daily teaching process, so as to exchange and study with peer experts.

\section{Acknowledgment}

Project source: shaanxi education science "13th five-year plan" project 2017 project: research on training mode of core competence of talents in engineering management of private universities (SGH17H483)

\section{References}

1. Liang Lijie. Architectural mechanics [M]. Beijing: China Electric Power Press, 2011.

2. Long Yuqiu et al. Structural Mechanics I-Basic Tutorial (3rd Edition). [M] Beijing: Higher
Education Press, 2012.

3. Lei Zhonghe. Structural Mechanics Learning Guidance [M].Beijing: Higher Education Press, 2005. 7.

4. Li Quanyong. A formula for the degree of freedom that can be generalized [J]. Journal of Guilin University of Electronic Technology, 1984, No. 1, 113-117.

5. Guo Weidong, Yu Jingjun. A New Method for Calculating the Degree of Freedom of Planar Mechanisms [J], Journal of Mechanical Engineering, 2013, 49(7): 125-129.

6. Zhang Junde. The establishment of the second type of computational degrees of freedom for the pole system, 1986, Issue 4, 55-56.

7. Zhou Qinghua, Zhang Yuxian. Study on Calculation of Degree of Freedom of Planar Mechanism [J], Coal Mining Machinery, 2005(4): 45-46.

8. Huang Shixin. On Calculation of Organizational Degree of Freedom [J]. Journal of Adult Education College of Hebei University of Technology, 2002, 17(2): 24-26.

9. Ouyang Fu, Liu Yanhua, Sun Dongmin. Exploration of Re-establishing the Calculation Formula of Degree of Freedom of Space Agency [J], 2003, 39(1): 60-64.

10. Cai Hanzhong, Ouyang Fu, Liao Mingjun. Research on the Application of Computational Formulas for the New and Old Degrees of Freedom of Institutions [J], 2011, 22(2): 35-38,87. 